Please do not remove this page

RMIT

UNIVERSITY

\title{
Advanced solutions for quality-oriented multimedia broadcasting
}

Muntean, Gabriel-Miro; Ghinea, Gheorghita; Frossard, Pascal; Etoh, Minoru; Speranza, Filippo; Wu, Hong Ren

https://researchrepository.rmit.edu.au/esploro/outputs/9921863957801341/filesAndLinks?institution=61RMIT_INST\&index=null

Muntean, G.-M., Ghinea, G., Frossard, P., Etoh, M., Speranza, F., \& Wu, H. R. (2008). Advanced solutions for quality-oriented multimedia broadcasting. IEEE Transactions on Broadcasting, 54(3, Part 2), 494-498.

https://doi.org/10.1109/TBC.2008.2004258

Published Version: https://doi.org/10.1109/TBC.2008.2004258

Repository homepage: https://researchrepository.rmit.edu.au

(c) 2008 IEEE. Personal use of this material is permitted. However, permission to reprint/republish this material for advertising or promotional purposes or for creating new collective works for resale or redistribution to servers or lists, or to reuse any copyrighted component of this work in other works must be obtained from the IEEE.

Downloaded On 2023/04/26 23:32:46 +1000 


\section{Advanced Solutions for Quality-Oriented Multimedia Broadcasting}

$\mathbf{M}$ ULTIMEDIA content is increasingly being delivered via different types of networks to viewers in a variety of locations and contexts using a variety of devices. The ubiquitous nature of multimedia services comes at a cost, however. The successful delivery of multimedia services will require overcoming numerous technological challenges many of which have a direct effect on the quality of the multimedia experience. For example, due to dynamically changing requirements and networking conditions, the delivery of multimedia content has traditionally adopted a best effort approach. However, this approach has often led to the end-user perceived quality of multimedia-based services being negatively affected. Yet the quality of multimedia content is a vital issue for the continued acceptance and proliferation of these services. Indeed, end-users are becoming increasingly quality-aware in their expectations of multimedia experience and demand an ever-widening spectrum of rich multimedia-based services.

As a consequence, there is a continuous and extensive research effort, by both industry and academia, to find solutions for improving the quality of multimedia content delivered to the users; as well, international standards bodies, such as the International Telecommunication Union (ITU), are renewing their effort on the standardization of multimedia technologies.

There are very different directions in which research has attempted to find solutions in order to improve the quality of the rich media content delivered over various network types. It is in this context that this special issue on broadcast multimedia quality of the IEEE Transactions on Broadcasting illustrates some of these avenues and presents some of the most significant research results obtained by various teams of researchers from many countries. This special issue provides an example, albeit inevitably limited, of the richness and breath of the current research on multimedia broadcasting services. The research issues addressed in this special issue include, among others, factors that influence user perceived quality, encoding-related quality assessment and control, transmission and coverage-based solutions and objective quality measurements.

\section{Solutions to Reduce the Negative EfFects of MulTiMEDIA DELIVERY OVER NETWORKS}

Authors Huang and Kuo in "Optimization of Hybridized Error Concealment for H.264" propose a novel Error Concealment method for H.264/AVC, using not only spatially and temporally correlated information but also the tandem utilization of two new coding tools: directional spatial prediction for intra coding and variable block size motion compensation of H.264/AVC.

Digital Object Identifier 10.1109/TBC.2008.2004258
Lin, Ke, Shieh, Chilamkurti and Hwang in the paper entitled "A RED-FEC Mechanism for Video Transmission over WLANs" propose a novel solution to improve the quality of video delivered over WLANs. Unlike previous AFEC schemes in which the FEC rate is determined on the basis of feedback information supplied by the receiver side, an appropriate FEC rate is determined by the wireless access point based upon an assessment of the current network traffic load. In comparison with previous solutions, the proposed scheme brings significant performance improvements without injecting an excessive number of redundant packets into the network.

\section{INTELLIGENT SOLUtions to INCREASE USER PERCEIVED Quality When Delivering Multimedia CONTENT OVER VARIOUS NETWORK TYPES}

Coudoux, Gazalet, Goudemand, Corlay, and Gharbi in their paper entitled "Extended Coverage for DSL Video Distribution Using a Quality-Oriented JSCC Architecture" introduce a new scheme to extend the coverage area for DSL video delivery while maintaining end-user perceived quality. The proposed architecture is based on a joint source-channel coding approach, and combines layered video transcoding with unequal error protection using hierarchical multi-carrier transmission. A new image quality metric is used to determine the optimal system parameters automatically in terms of end-user perceived quality.

In "Laboratory Measurement Campaign of DVB-T Signal with Transmit Delay Diversity", Di Bari, Bard, Zhang, Nasr, Cosmas, Loo, Nilavalan, Shirazi and Krishnapillai, on the other hand, examine the performance of transmission delay diversity (DD) with two antennas in order to improve the reception of DVB-T/H systems operating in different realistic propagation conditions. Their research shows that the quality of reception is significantly improved by transmission delay diversity, especially in fast fading mobile broadcasting applications.

Baldi, De Martin, Masala and Vesco present in "Quality-oriented Video Transmission with Pipeline Forwarding" a framework for optimizing the delivery of video data over packet networks. This paper considers the perceptual relevance of video data for pipeline forward and proposes two new scheduling algorithms. Extensive analysis and simulation results demonstrate the benefit of the proposed approach while also investigating the impact of the encoding scheme on the overall solution performance.

\section{DELIVERY PERFORMANCE}

In the invited paper "Coalition-based Resource Reciprocation Strategies for P2P Multimedia Broadcasting" Park and van der Schaar concentrate on chunk-based data-driven multimedia peer-to-peer broadcasting. The authors propose a peer matching 
algorithm that discriminates multimedia peers based on their attributes. Following a matching process, peers create coalitions with selected peers with which they share their multimedia data chunks. The paper analyses the peer benefit by making coalitions and assesses the impact that their interactions have on the overall system quality.

In the paper "Analysis of DVB-H Network Coverage with the Application of Transmit Diversity" Zhang, Zhang, Cosmas, Loo, Owens, Di Bari, Lostenlan and Bard investigate DVB-H network coverage with the application of Cyclic Delay Diversity (CDD) during transmission. The benefits and effectiveness of CDD are demonstrated through simulations of several scenarios of coverage in DVB-H networks. The channel model used in the simulations is based on COST207.

Jaime, Leao, Silva and de Marca in the paper "User Level fairness of a Multi-rate $3 G$ Technology (EV-DO) with Application to Digital TV Return Channel Technology" investigate the end-to-end performance of individual users when a particular multi-rate $3 \mathrm{G}$ wireless air interface is adopted for delivering digital TV services enhanced with a return channel. The authors show that a simple solution can greatly improve the fairness characteristics without sacrificing the overall system throughput.

Plets, Joseph, Verloock, Tanghe, Martens, Deventer and Gauderis propose in their paper "Influence of Reception Condition, MPE-FEC Rate and Modulation Scheme on Performance of $D V B-H$ " a method for evaluating the performance of DVB-H networks in practical situations. The evaluation process considers varying MPE-FEC rates and modulating schemes at various physical locations for indoor, outdoor and mobile receptions. Performance measure is taken in terms of the percentage of valid reception, MPE-FEC gains, carrier to interference-plus-noise ratios, and minimal signal strengths.

\section{Quality Assurance Through Admission CONTROL}

Tackling the issue of admission control, Davy, Jennings and Dmitri Botvich present in their paper "Revenue Optimized IPTV Admission Control using Empirical Effective Bandwidth Estimation" an algorithm for maximizing IPTV revenue based on an empirical estimation of effective bandwidth. Using real traffic traces, the authors show that their proposed algorithm does maintain high bandwidth utilization, with minimal violations of the QoS of IPTV video.

In the paper entitled "Call Admission Control for Aggregate MPEG-2 Traffic over Multimedia Geo-Satellite Networks", De Rango, Tropea, Fazio and Marano investigate statistical multiplexing based on discrete bandwidth (SMDB) levels of group of picture rates, providing a call admission control algorithm that is tailored specifically for MPEG streams. For MPEG sources, the SMDB achieves higher utilization rate than the statistical multiplexing based on the normal/log-normal distribution method with better approximation of the MPEG traffic characteristics.

\section{EFFECT ON USER PERCEIVED QUALITY}

Younkin and Corriveau in "Determining the Amount of Audio-Video Synchronization Errors Perceptible to the Average End-User" present a study in which a psychophysical methodology (staircase method) was used to determine the minimum amount of lip synchronization error, i.e., audio-visual synchronization error, which can be detected by end-users during multimedia delivery.

Shmueli, Hadar, Huber, Maltz and Huber in "Effects of the Encoding Scheme on Perceived Video Quality Transmitted over Lossy Internet Protocol Networks" present the results of a subjective test performed on a large group of subjects. The authors have analysed user perceived quality when video streams with varying spatial and temporal activity levels as well as different levels of contrast and colorfulness are delivered over lossy best effort networks. The paper presents a novel solution which suggests an encoding scheme and a content adaptation mechanism to best perform in existing network conditions and to best suit video sequence characteristics.

Huynh-Thu and Ghanbari in their paper "Temporal Aspect of Perceived Quality in Mobile Video Broadcasting" present a set of subjective quality assessment experiments conducted to measure the impact of temporal artifacts on video quality and characterize the influence of content motion on perceived quality. Human subjects' responses to jerkiness and jitter by considering different levels of strength, duration and distribution of the temporal impairments were assessed when using video sequences with different characteristics. The results presented in this paper provide useful information for the development of an objective video quality metric.

Authors Monteiro, Calafate and Nunes in "Evaluation of the H.264 Scalable Video Coding in Error Prone IP Networks" provide an analysis and evaluation of the scalable extension of the H.264/AVC video coding standard called Scalable Video Coding (SVC) when transmitting in error prone environments. Given the interdependency structure in an SVC encoded video, the analysis tries to quantify the degradation caused by packet losses in the received and decoded video.

\section{QUALITY METRICS}

Winkler and Mohandas review the evolution of video quality measurement techniques and the current state-of-the-art in the invited paper entitled "The Evolution of Video Quality Measurement: From PSNR to Hybrid Metrics". In particular, the paper discusses the various types of objective quality metrics and their uses in different application scenarios. The paper further introduces a "hybrid" metric, VFactor that uses both transport- and bitstream-information in video communication applications. Finally, the paper reports the main standardization activities in video quality assessment, and discusses the emerging trends in the area.

Gunawan and Ganbari in their paper entitled "An Efficient Reduced-reference Video Quality Meter" present a reduced-reference video quality assessment method. Based on the local harmonic strength feature, the proposed method is effective for continuous monitoring of video quality and produces good results without significantly degrading the performance of the video quality model.

Yang, Huang, Nguyen, Guest, and Das propose in the paper "A New Objective Quality Metric for Frame Interpolation Used in Video Compression" a new method for assessing the spatial quality of the temporally interpolated frames. The proposed metric quantifies the severities of several pronounced interpola- 
tion artifacts and combines them along with human visual factors to form a global (i.e., frame-wide) quality measurement. It further considers additional quality impairment caused by locally aggregated distortion. The new metric is shown to outperform other commonly used metrics in subjective tests.

Eden in the paper "No-Reference Image Quality Analysis for Compressed Video Sequence" presents a measurement of image spatial activity (taken as an indicator of visual masking) and discusses how it relates to the visibility of compression artifacts in natural still images. This relation is investigated through a subjective assessment experiment which shows a strong correlation between the visibility of compression artifacts and the spatial activity of the images. This information is used to derive a "perceptual" PSNR, or pPSNR for short, which can be used as a basis for further no-reference PSNR quality measurements.

\section{ENCODING-RELATED QUALITY ASSESSMENT AND CONTROL}

In the paper entitled "Traffic and Quality Characterization of Single-Layer Video Streams Encoded with the H.264/MPEG-4 Advanced Video Coding Standard and Scalable Video Coding Extension", van der Auwera, David, and Reisslein analyse the rate-distortion characteristics of the streams generated by the recent video compression standards. The proposed analysis shows that the H.264/AVC codec and SVC extension achieve lower average bit rates than MPEG-4 Part 2 video streams, at the expense, however, of significantly increased traffic variabilities that remain at a high level even with smoothing. This potentially induces increased frame losses and important rate-distortion/ rate-variability/ encoding complexity tradeoffs in networked application scenarios.

The paper "Three Dimensional Scalable Video Adaptation via User-end Perceptual Quality Assessment" authored by Zhai, Cai, Lin, Yang and Zhang introduces a way to select spatial, temporal and SNR resolutions for scalable video based on content specific metric such as blockiness, blur, and motion jerkiness, considering the human visual system, rather than the bit-rate alone. For such purpose, a low-complexity algorithm for mobile terminals is developed and its effectiveness is tested through experiments presented in this paper.

\author{
Gabriel-Miro Muntean, Guest Editor \\ Performance Engineering Laboratory \\ Dublin City University \\ Glasnevin, Dublin 9, Ireland \\ (e-mail: munteang@eeng.dcu.ie) \\ GHeORGHITA GHINEA, Guest Editor \\ School of Information System, Computing \\ and Mathematics \\ Brunel University \\ Uxbridge UB8 3PH, U.K. \\ (e-mail: george.ghinea@brunel.ac.uk) \\ PASCAL Frossard, Guest Editor \\ Signal Processing Laboratory \\ Ecole Polytechnique Fédérale de Lausanne (EPFL) \\ Lausanne 1015, Switzerland \\ (e-mail: pascal.frossard@epfl.ch) \\ MinORU ETOH, Guest Editor \\ NTT DoCoMo Research Laboratories \\ Yokosuka 239-8536, Japan \\ (e-mail: etoh@ieee.org)
}

FILIPPO SPERANZA, Guest Editor

Communications Research Center

Ottawa ON K2H 8S2, Canada

(e-mail: Filippo.Speranza@crc.ca)

Hong ReN Wu, Guest Editor

Royal Melbourne Institute of Technology

Melbourne VIC 3000, Australia

(e-mail: henry.wu@rmit.edu.au)

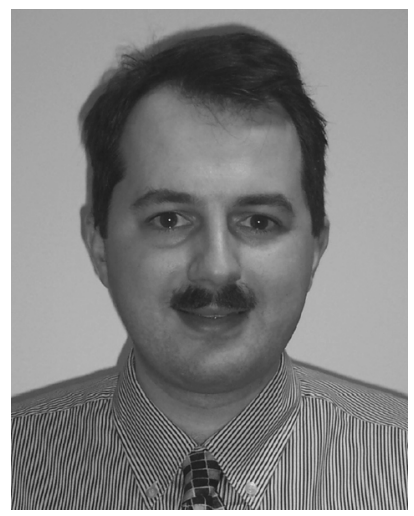

Gabriel-Miro Muntean is a Lecturer with the School of Electronic Engineering and co-Director of the Performance Engineering Laboratory at Dublin City University, Ireland. He obtained his Ph.D. degree from Dublin City University, Ireland for research in quality-oriented adaptive multimedia streaming over wired networks in 2003. He was awarded the B.Eng. and M.Sc. degrees in Software Engineering from the Computer Science Department, "Politehnica" University of Timisoara, Romania in 1996 and 1997 respectively. Dr. Muntean's research interests include quality and performance-related issues of adaptive multimedia streaming, and personalized eLearning over wired and wireless networks and with various devices. Dr. Muntean has published over 70 papers in top-level international conferences and journals, as well as an authored book, two edited books and five book chapters. Dr. Muntean is Associate Editor with the IEEE TRANSACTIONS ON BROADCASTING and reviewer for important international journals, conferences and funding agencies. He is member of IEEE and Research Institute for Networks and Communications Engineering (RINCE) Ireland. 


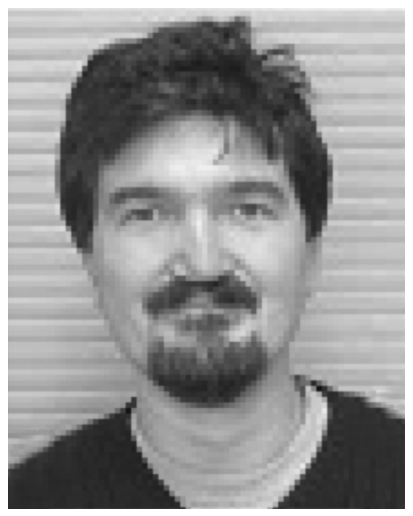

Gheorghita Ghinea (M'02) received the B.Sc. and B.Sc. (Hons) degrees in computer science and mathematics, in 1993 and 1994, respectively, and the M.Sc. degree in computer science, in 1996, from the University of the Witwatersrand, Johannesburg, South Africa; he then received the Ph.D. degree in Computer Science from the University of Reading, United Kingdom, in 2000. He is a Reader in the School of Information Systems and Computing at Brunel University, United Kingdom. Dr. Ghinea has over 60 international refereed publications and regularly consults for public and private organizations in his area of expertise. His research interests span perpetual aspects of multimedia, Quality of Service and multimedia resource allocation, as well as computer networking and security issues.

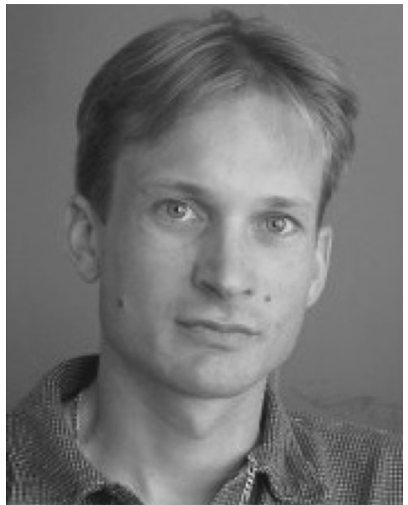

Pascal Frossard (S'96-M'01-SM'04) received the M.S. and Ph.D. degrees, both in electrical engineering, from the Swiss Federal Institute of Technology (EPFL), Lausanne, Switzerland, in 1997 and 2000, respectively. Between 2001 and 2003, he was a member of the research staff at the IBM T. J. Watson Research Center, Yorktown Heights, NY, where he worked on media coding and streaming technologies. Since 2003, he has been an assistant professor at EPFL, where he heads the Signal Processing Laboratory (LTS4). His research interests include image representation and coding, nonlinear representations, visual information analysis, joint source and channel coding, multimedia communications, and multimedia content distribution. Dr. Frossard has been the General Chair of IEEE ICME 2002 and Packet Video 2007, and a member of the organizing or technical program committees of numerous conferences. He has been an Associate Editor of the IEEE TRANSACTIONS ON MULTIMEDIA (2004-) and of the IEEE TRANSACTIONS ON CIRCUITS AND Systems FOR VIDEO TECHNOLOGY (2006-). He is an elected member of the IEEE Image and Multidimensional Signal Processing Technical Committee (2007-), the IEEE Visual Signal Processing and Communications Technical Committee (2006-), and the IEEE Multimedia Systems and Applications Technical Committee (2005-). He has served as Vice-Chair of the IEEE Multimedia Communications Technical Committee (2004-2006) and as a member of the IEEE Multimedia Signal Processing Technical Committee (2004-2007). He received the Swiss NSF Professorship Award in 2003 and the IBM Faculty Award in 2005.

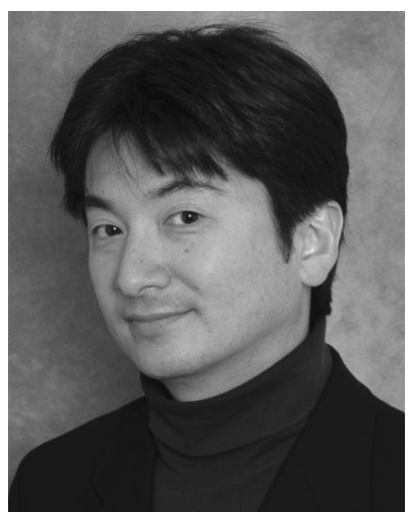

Minoru Etoh is Deputy Managing Director of Research Laboratories at NTT DoCoMo, Japan. In 90's, he was leading an image communication research team in Matsushita Electric and participated in MPEG-4 standardization. He joined Multimedia Laboratories of NTT DoCoMo in 2000, where he contributed to launching DoCoMo's 3G mobile multimedia services including video phones and audio-visual content download applications. He was also appointed at Managing Director of DoCoMo USA Labs in 2002 and Multimedia Labs in 2005 respectively. He served as visiting professor of Nara Institute of Science and Technology in 2003-2006, and is still teaching mobile communication technologies in Osaka University. His expertise a wide range of mobile multimedia: network architecture, terminal software, coding technologies, media transport, and data mining. He received his B.E. and M.S.E.E. from Hiroshima University, Ph.D. degree from Osaka University, in 1983, 1985 and 1993 respectively. 

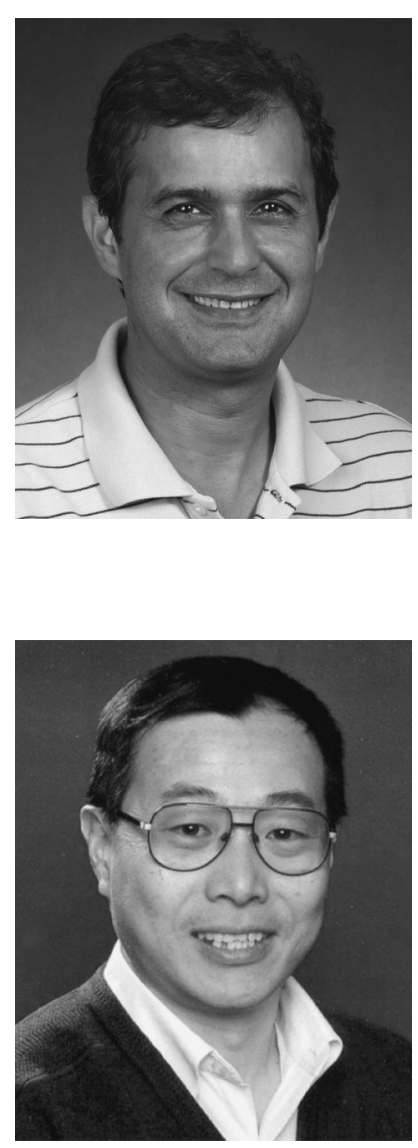

Hong Ren Wu received his B. Eng. and M.Eng. from the University of Science and Technology, Beijing (formerly Beijing University of Iron and Steel Technology), P.R. China, in 1982 and 1985, respectively. He received his Ph.D in Electrical and Computer Engineering from the University of Wollongong, N.S.W. Australia, in 1990. Dr. Wu worked as academic staff in Chisholm Institute of Technology and then Monash University, Melbourne, Australia from April 1990 to January 2005; last as an Associate Professor in Digital Systems. Dr. Wu has been with Royal Melbourne Institute of Technology, Australia, since February 2005, as Professor of Visual Communications Engineering and Discipline Head, Computer and Network Engineering in the School of Electrical and Computer Engineering. His research interests include fast DSP algorithms, digital picture compression and quality assessment, video processing and enhancement, embedded DSP systems and their industrial applications. His most recent publications include a book co-edited with Prof. K.R. Rao of University of Texas at Arlington on Digital Video Image Quality and Perceptual Coding. 